\title{
A Baseline for a School Management Information System
}

\author{
Alan Strickley \\ CRIA Technologies; Harborne, Birmingham, England \\ alan.strickley@gmail.com
}

\begin{abstract}
This paper examines previous studies and supplier recommendations for the requirements of Management Information Systems (MISs) in schools. Utilising the broad headings in the Becta (2006) document 'functional requirements for information systems including MIS' it expands on its recommendations as a baseline for a school's MIS. It looks in brief at 34 different areas that may form part of a school's system and considers the minimum features that each should encompass. It considers the need for more detailed examination of some of the categories and concludes that much has been achieved over the last 20 years or so. However it also notes that the areas of open standards, interoperability, transfer, functionality, cost effectiveness, statutory driven developments and usability are still major issues.
\end{abstract}

Keywords: MIS; Management; Information; System; Schools; Interoperability; Framework; Software.

\section{Introduction}

There have been many previous studies into the use of MISs in schools including; Strickley, 2009a \& 2004; Baker et al, 2008; Fung et al, 1998; Ip Tsang \& Lee, 1997; Fung \& Ledesma, 2001a\&b; Visscher \& Bloemen, 2001; Nolan et al, 1996; Nolan \& Lambert, 2001; Nolan \& Ayres, 1996; Wild \& Walker, 2001; Visscher, Wild \& Smith, 2003; Selwood, 1995, 2004 \& 2005; O'Mahony, 1998; Barta, Telem \& Gev, 1995. Many of them have made recommendations as to the minimum and preferred requirements of a school MIS.

In addition the various school MIS suppliers provide information on the specifications and advantages of their own specific systems including Capita SIMS, (Capita, 2009), Serco CMIS, (SERCO, 2009), Wauton Samuel PASAPP, (Wauton Samuel, 2009), Bromcom, MIS, (Bromcom, 2009), Research Machines Integris, (Research Machines, 2009), ScholarPak (ScholarPak, 2009) and Schoolbacs (Schoolbacs, 2009).

This paper looks at previous research, the MIS suppliers' information, The 'Information Management Strategy Framework' (Becta, 2008), The 'School Management Information Systems and Value for Money' report (Becta, 2005b) and 'What to look for in a management information system' (Becta, 2005a). Using the Becta (2006) framework as a template it expands on each of the categories contained within it adding new ones as appropriate. 
The paper makes no assumption that the MIS is from a single supplier (although it could be) but that all of the modules should work in an interoperable way sharing common data with each other and under a single over-arching data management and security protocol. It considers the main users will be administrators, teachers, parents, pupils and the senior management team and that although the requirements of primary and secondary schools may be different this should not be assumed. Whilst most of the attributes described would be applicable to any school MIS many of the legal references apply to schools in England only.

The following sections follow those in the Becta (2006) report with extra sections added at the end. The order of the sections is not considered to have any significance to their importance.

\section{Specification}

\subsection{Access Remotely}

Multiple site access to the MIS is critical to its use and usefulness (section 2.2). As Strickley (2009a) found in his study of primary schools many teachers, parents and governors do not have electronic access to even the most basic data stored on the school MIS and often have to rely on, at best, hard copy.

In addition the availability of remote access outside of the school building is important for several reasons:

- Lack of non-contact time in school to use the system (particularly for primary schools where there is less non-contact time).

- Lack of access points within the school to accommodate all staff.

- Lack of access at point of source for data input.

In order to achieve this full availability the MIS must have the capability of being accessed from any suitable hardware, at any location, through the internet using an eportal or similar. As a result the transfer of data must be secure and the access point subject to strict password controls.

The use of secure wireless technology within the school should help alleviate some of the issues of school access points. However, there is still the need for hardware that can communicate wirelessly together with suitable work areas that comply with health and safety guidelines for computer workstations (HMSO, 1992).

It should not be assumed that internet connectivity is always available (or even at all) and the ability to work offline and upload when connected should be a feature of the system where appropriate.

In short the MIS should be available, with appropriate restrictions and security, at any place at any time accessible through a variety of hardware options.

\subsection{Accessibility}

The system must be accessible through an intuitive Human Computer Interface (HCI; see section 2.30) and support those with disabilities through Disability Discrimination 
Act (DDA) compliance (HMSO, 2005a) and the Web Accessibility Initiative (WAI, 2009). Screens should be customisable to individual requirements where possible e.g. text size, colour, language etc.

As Strickley (2009a) suggests many of the current MISs are not designed with the user in mind and many web-enabled options are identical in layout to the non-web version. The different requirements and modes of use of the different access channels must be part of the design.

\subsection{Admissions}

Admissions for first entry (reception), secondary transfer and in year or casual admissions are all important transfer stages for pupils (Strickley \& Allen, 2008). It is therefore critical that the pupil profile is transferred when the pupil moves school. Whilst the Common Transfer File (CTF) (DSCF 2009a) and the more specific Admissions Transfer File (ATF) (DCSF, 2009b) achieve this to a limited extent they suffers from a lack of rich pupil data and a difficult transfer process through the s2s secure transfer site (DCSF 2009c).

The ability to seamlessly import new admissions data into an MIS is essential for the continued learning of the pupil with the added advantage of reducing data input into the system for administrative staff.

For the increasing number of schools that administer their own admissions policy (academies; voluntary aided and foundation schools) the MIS should be capable of importing the appropriate admissions application data (Admissions Data Transfer File; ADT) ranking the pupils as defined in the school's over-subscription criteria and sending the file back as an Admissions School List (ASL) file to the home Local Authority (LA) for processing (DCSF, 2009b). This again should reduce data input burdens for schools and LAs as well as ensuring that the admissions criteria of the school are being applied fairly and consistently (DCSF 2009d).

The above own admitting authority schools will also need to keep records of appeals by parents who have failed to secure a place at the school as part of the statutory reporting requirements for school census (DCSF 2009e). Also see section 2.29.

\subsection{Assessment Management}

Assessment is key to the school as a measure of its effectiveness, the performance of its pupils and the development and improvement of its learning and teaching.

The assessment software should provide all of the flexibility and usability of the teachers' traditional mark book with the added functionality of a software tool which will support analysis, predicting, standardisation and statistical modelling through an intuitive interface.

As the Becta (2006) document suggests all tests should be recorded in the system. However the results of these tests should be available on a need to see basis to ensure the confidence and support of the teaching staff entering them.

The process of recording should enable the administration of statutory, internal and external exams and tests. These results should also be accessible to parents, in the 
appropriate format, as part of the online reporting process (section 2.34). This will also reduce data input burdens. The ability to add data at the point of creation whether online or offline is particularly important in this area.

Any Integrated Learning Systems (ILSs) and learning platform should integrate seamlessly with this module with assessment results being recorded automatically.

\subsection{Attendance}

The collection and transfer of attendance data is a statutory requirement (DCSF, 2006) for schools and therefore a method of easily collecting this data and transferring it is an essential statutory element of a school's MIS. In addition the continued and regular attendance of pupils is critical to their educational achievement and the identification and intervention of poor attending pupils is a vital tool in school improvement.

Seamless capture here is the key, at source, via a PDA, laptop, swipe-card or similar. The ability to easily capture lesson by lesson attendance as well as am-pm registration is also an added advantage particularly for secondary schools. In this latter respect the module needs to work in tandem with the timetabling and options modules.

As Strickley (2009a) suggests attendance reporting should be real-time via an alerts system or reporting tool and not just used for statistical reports at the end of the day, week or term.

The software should enable the proactive monitoring of attendance patterns, alert appropriate staff to unusual activity and activate intervention when key critical points are reached.

\subsection{Authentication}

A school's MIS will contain a large amount of sensitive data about children, parents, teachers and other associated personnel. Clearly such a database needs robust authentication before users can access any part of it and in addition strict business rules must be applied that control the exact data items that may be accessed.

Access to the system should be at a minimum through user ID and strong, frequently changed, password with additional use of biometrics an option if appropriate.

Wireless, intranet, internet and LAN authentication protocols must be in place and regularly monitored to ensure that only approved personnel are accessing the system.

\subsection{Behaviour Management}

Both positive and negative aspects of behaviour should be recordable on the system. The system should allow the recording of action taken and outcomes. See section 2.31 for exclusions.

Reports should be available to all appropriate school staff in real-time and analysis tools should be capable of illustrating trends and variables as appropriate. Event 
driven alerts should be used to inform staff of critical issues such as specific lesson/teacher issues, repeat occurrences, items for weekly newsletter etc.

It is important that the module is used for both positive and negative aspects and not just for negative incidents. Staff training will be required to make sure that the system is used in a consistent and equitable way.

\subsection{Consistent Learner Information}

The system should ensure that there is minimal duplication of information and that processes should be automated to avoid errors and inconsistencies.

The use of consistent datasets will help to enable consistency and interoperability. All data should be validated against standard benchmarks such as BS7666 (Cabinet Office, 2009), Common Basic Data Set (CBDS), (DCSF, 2009f) etc. at source to ensure that consistency is maintained and that cross -system transfer is possible. The development of the Systems Interoperability Framework (SIFA, 2009) and the establishment of the Information Standards Board (ISB, 2009) may assist in this with a view to common data standards across all partners including health, social services, youth services etc.

\subsection{Data Protection}

Compliance with the Data Protection Act (DPA, HMSO, 1998) is clearly essential. Fair Processing Notices (FPNs, DCSF, 2009g), also known as privacy notices, should encompass all data sharing activities and should be generated for all data collection processes such as data collection sheets, emails, web forms etc.

The modules within the MIS should comply with the security guidelines in the Becta (2009) report.

\subsection{Information Access}

Edit, view and restricted access roles should be available and applied to all fields including User Defined Fields (UDFs). Predefined groups should not be assigned as a default. Access rights should be at field level.

These access rights should be reflected in the fields accessible in the reporting module (section 2.13).

\subsection{Learner Information Exchange}

The system should comply with the statutory requirements of pupil data transfer as detailed in HMSO (2005b) using the CTF. It should comply with the data collections from the various statutory bodies such as DCSF, QCA etc.

This statutory baseline should be extendable for more rich data about the pupil for transfer to other systems (including those outside of the school system such as colleges) as detailed in (Strickley \& Allen, 2007). 
The system should also support the use of Unique Learner Numbers (ULNs, MIAP, 2009a) for the transfer of information through Managing Information Across Partners (MIAP) learner registration service.

\subsection{Messaging}

The MIS should act as a conduit for the creation, transmission and receipt of all types of messages.

These may be telephone voice messages received via any telemetry system and captured by the system; emails from inside or outside of the school intranet; SMS texts sent to an approved school mobile number; emails sent via a web-based email system for parents; short messages sent via the web portal; instant messaging; digitised documents and any other approved method of contact.

Essentially the system should be inclusive to all messaging to and from the school making it unnecessary to move from application to application to access multichannel information and thus reducing the risk of missing vital information.

Contact information needs to be prioritised to prevent what could amount to spamming and the creation of groups with similar profiles should be simple to achieve.

\subsection{Reporting}

An MIS is only as good as the information that can be extracted from it. Ease of use and functionality are key. Simple reports should be menu-based and pre-defined for the users, whilst reports of intermediate complexity should be menu driven or use "wizards" enabling most users to utilise with minimal training. The development of complex reports should be available through a simple programming interface.

Reporting should take place across modules employing the same back-end database and should also be able to report across different databases providing the appropriate keys are available.

Whilst some of the simpler pre-defined reports may be modular based an overarching reporting tool must be available for the more complex requirements. A third party tool may well be the most appropriate and effective solution.

Internal and statutory reports should emulate the same information access security protocols as for direct access to the MIS and should not enable access via stealth.

All types of report should be available from simple list reports to more complex mail merges, cross tab reports and graphs and should be available for publication in a variety of formats e.g. pdf; doc and rtf.

\subsection{Special Educational Needs (SEN)}

The MIS must cover the statutory (DfES, 2001) administration and reporting functions that are required by the law. In addition it should enable the efficient and effective management of the education and well-being of SEN pupils by assisting practitioners to develop learning/education plans, assimilate progress and past achievements and monitor and report on progress at a micro level (section 2.34). 


\subsection{Data Returns}

The system must be capable of generating all of the central and local government returns/census in an efficient manner utilising data held on the system to the full . Error checking and validation should be intuitive and assist in cleaning the data held on the system to the advantage of the school. The format of reports should be in a variety of interoperable formats.

\subsection{Support}

The system should be supported through online help, supplier helpdesk, email, telephone and local support unit. Help should be intuitive and available when required but unobtrusive. System upgrades should be accompanied by appropriate explanatory materials including changes $\log$ and at regular intervals without excessive software patches applied at a later date.

\subsection{Timetable Management}

The timetable is a complex and significant process particularly for a large secondary school. The timetable module should support all of the modelling functionality required including rooms; times; locations; special needs access; travel time; nonstandard timetable cycles etc. It should not be seen as an annual one-off event and should be accessible by staff, parents and pupils in a cut down format through the network and web portal for reference, cover and general information.

\subsection{Workforce Management}

The system should enable the management of personnel records, job evaluation, pay scales, training and development, attendance, sickness etc. enabling the generation of statutory returns (DCSF, 2009h) and linking with financial management to enable a full business model of the school and its staff.

Such a sensitive area should have the highest security protocols in place.

\subsection{Asset Management}

The MIS should be capable of recording and tracking all assets in the school including consumables. The normal reporting and alerting functions should be available to enable good stock management.

\subsection{Coursework Management}

The system should support the storage, retrieval, classification and archiving of pupils' coursework materials. These may be those as submitted for examination or pupils' work as part of their day-to-day education or after-school activities. Digitisation of the materials together with metadata for identification must be available. Where appropriate the facility to transfer outside of the school system (sections $2.33 \& 2.27$ ) to external examiners, employers or other schools must be available and efficiently implemented. 


\subsection{Curriculum Planning}

The system must be capable of storing the overall school development plan in an appropriate format. It must cater for all levels from whole-school, through departmental to teacher lesson plans. It must also hold resources required and enable forecasting and modelling for future planning with appropriate links to finance, asset management and assessment etc.

\subsection{Dinner Money}

The recording of dinner, trip, school fund etc. monies should be a feature of the MIS. The process should be linked to the school's financial system with full audit trails and authentication. The system should be linked to any cashless catering systems and ipayment facility and should be capable of printing receipts, remittances and cheques as appropriate.

\subsection{Document Management}

The system needs to be capable of the storage, retrieval, classification and archiving of documents including full metadata. Digitisation may be through a variety of methods but the system should be capable of importing and exporting all standard formats.

\subsection{Financial Management}

Full, partial or limited financial management, to industry standards, should be available capable of being dovetailed with existing over-arching systems from the Local Authority (LA) or other systems. The system should accept all payments including those via the internet and enable all forms of ordering across the school with appropriate access levels (section 2.9).

Full audit trails and security protocols must be in place.

\subsection{Library}

The MIS should have the option to run a fully functional library system including reference, lending, borrowing, returns, reminders and fines system. It should also be available online and utilise electronic copies where appropriate. The system should be adaptable for all types of schools.

\subsection{Options}

The facility to accommodate options, particularly for years 9 to 11 , to facilitate curriculum planning and timetabling should be incorporated into the MIS with the additional functionality to handle diplomas and non-standard requirements.

\subsection{Transition Portfolios}

An ePortfolio enables learners to have a record of their progress throughout their lifelong learning (Strickley, 2009b). The creation, maintenance, access and seamless transfer of ePortfolios should be a feature of the MIS (sections 2.11, 2.20 and 2.34). 


\subsection{Web Services}

The system should incorporate interoperability standards, such as SIF (2009) that enable seamless data exchange to occur between modules within the MIS and appropriate systems outside of it. This should be through the use of an Application Programming Interface (API) or other appropriate protocol e.g. xml (section 2.33).

\subsection{Appeals}

The MIS should be capable of recording, administrating and reporting on appeals where the school undertakes this function as a foundation, voluntary aided school or academy (section 2.3).

\subsection{Human Computer Interface (HCI)}

The MIS modules should all incorporate HCI best practice (COI, 2009) and should have a similar look and feel between each module using a consistent GUI and shortcut keys (section 2.2).

\subsection{Exclusions}

The MIS should enable the recording, retrieval and internal and external statutory reporting of exclusions information (DCSF, 2009i). Alerts should be in place to warn when statutory limits are approaching and it should be capable of modification outside of the statutory framework for internal purposes.

\subsection{Alerts}

Alerts are a critical part of an MIS. They must be easy to set up and flexible in terms of recipients, mode of receipt, activation levels and complexity of initiation thresholds. For example they should be able to alert a predefined group by email and SMS, if a mobile number is available, when a combination of criteria are reached.

\subsection{Transfers}

As referred to in sections $2.3,2.11$ and 2.27 the ability to transfer the data between modules within a school, between schools, to the LA and other agencies such as DCSF and QCA is a critical feature of an MIS. This will require a more open standards approach such as that proposed by ISB and SIF (see section 2.8 and 2.28).

\subsection{Reports to Parents}

The statutory requirement for secondary schools in 2010 and primary schools in 2012 (DCSF, 2009j) to enable electronic reporting of attendance and behaviour (both positive and challenging) progress and attainment and SEN information to parents is a critical mover to increase access and engagement for parents (Strickley, 2009). The ability to record and share this information will need to be embedded in all MISs by the dates above. 


\section{Conclusions}

There is no doubt that school MISs have improved greatly over the last 20 years and most incorporate, to some extent, the functions described in this paper.

However there are still some major issues which need to be addressed:

- There is still a tendency for MISs to be marketed as a single complete solution. Whilst many of the major suppliers offer all or most of the functionality a school might need there is little opportunity to mix and match the modules between suppliers. This is as a result of the lack of open and interoperable standards being used by the suppliers as discussed in detail in Becta (2005).

- The lack of transfer between different schools (and LA) systems is highlighted in Strickley \& Allen (2008). Transfer needs to seamless and capable of transferring all pupil data as required.

- Support for school MISs needs to be flexible and cost effective particularly where different suppliers are involved (Becta 2005).

- Modules need to be more adaptable to the needs of the school be they secondary, primary, special, small or large.

- Whilst the requirements of statutory legislation are key functions of the MIS they must not be the drivers of the development of it.

Further work is under consideration to expand upon this paper to give more examples and details, with references to case studies. It will be interesting to see how systems compare against these criteria in five years from now.

\section{References}

Becta: What to look for in a management information system (2005a),

http://schools. Becta.org.uk/index . php? section=lv\&\&catcode= ss_lvstr_02\&rid=195 (accessed August 7, 2008)

Becta: School management information systems and value for money. Becta, Coventry (2005b)

Becta: Functional requirements for information management including MIS (2006),

http: //schools. Becta.org.uk/index.php? section=lv\&catcode= ss_lv_str_02\&rid=12915 (accessed August 7, 2008)

Becta: Information management strategy framework. Becta, Coventry (2008)

Becta: Data handling security guidance for schools (2009),

http: //schools. Becta.org.uk/index . php? section=lv\&catcode= ss_1v_saf_se_03\&rid=14734 (accessed November 03, 2009)

Baker, G., O’Mahony, C.D., Selwood, I.D., Strickley, A.: ITEM Evolution. In: Tatnall, A., Visscher, A., Finegan, A., O’Mahony, C. (eds.) Evolution of Information Technology in Educational Management, pp. X-X. Springer, Boston (2008)

Barta, B.Z., Telem, M., Gev, Y. (eds.): Information technology in educational management. Chapman \& Hall, London (1995)

Bromcom: Home Page (2009), http: / / www . bromcom. com/ (accessed October 7, 2009)

COI: COI Usability toolkit (2009), http: / / usability. coi.gov.uk/

(accessed October 7, 2009) 
Cabinet Office: BS7666 standard (2009),

http: / /www. cabinetoffice.gov.uk/govtalk/schemasstandards / e-gif/datastandards/address.aspx (accessed October 14, 2009)

Capita: SIMS MIS homepage (2009),

http: / /www. capitaes.co.uk/SIMS/sims . asp (accessed October 8, 2009)

DCSF: The Education (Pupil Registration) (England) Regulations. HMSO, Norwich (2006)

DCSF: The Common Transfer File (2009a),

http: / /www. teachernet.gov.uk/management/ims / datatransfers / CTF /

(accessed October 8, 2009)

DCSF: Admissions transfer files (2009b),

http: / / www . teachernet.gov.uk/management/ims/datatransfers /

coordadmissions/ (accessed October 8, 2009)

DCSF: s2s website secure transfer system $(2009 \mathrm{c})$,

http: / /www. teachernet.gov.uk/management/ims / datatransfers / CTF / (accessed October 8, 2009)

DCSF: School admissions code. HMSO, Norwich (2009d)

DCSF: School Census Definitions (2009e),

http: / /www. teachernet.gov.uk/management/ims/datacollections / (accessed October 14, 2009)

DCSF: Common Basic Data Set, CBDS (2009f),

http: / /www. teachernet.gov.uk/management/ims / datamanagement /

cbds / (accessed October 14, 2009)

DCSF: Fair Processing Notices (2009g),

http: / / www. teachernet.gov.uk/management/ims / datamanagement /

privacynotices / (accessed October 14, 2009)

DCSF: School Workforce Census (2009h),

http: / / www. teachernet.gov.uk/management/ims/archive/

arcdatacoll/swf/ (accessed October 14, 2009)

DCSF: Exclusion reporting (2009i),

http: / / www. teachernet.gov.uk/wholeschool/behaviour/exclusion/ relateddocs / (accessed October 14, 2009)

DCSF: Reporting to parents (2009j),

http: //www.teachernet.gov.uk/_doc/12680/Final\%20online\%

20 reporting\%201etter\%20 online. doc (accessed October 14, 2009)

DfES: Special Educational Needs: Code of practice. HMSO, Nottingham (2001)

Fung, A.C.W., Visscher, A.J., Wild, P., Selwood, I.D.: SAMS in Hong Kong school: The first evaluative findings of a large-scale implementation of school management information systems. In: Fulmer, C., Barta, B., Nolan, P. (eds.) The Integration of Information for Educational Management, pp. 19-38. Felicity Press, Whitefield (1998)

Fung, A.C.W., Ledesma, J.: SAMS in Hong Kong: A centrally developed SIS for primary and secondary schools. In: Visscher, A.J., Wild, P., Fung, A.C.W. (eds.) Information Technology in Educational Management, pp. 39-53. Kluwer, Netherlands (2001a)

Fung, A.C.W., Ledesma, J.: SAMS in Hong Kong: A user acceptance audit. In: Nolan, C.J.P., Fung, A.C.W., Brown, M.A. (eds.) Pathways to Institutional Improvement with Information Technology in Educational Management, pp. 121-145. Kluwer Academic Publishers, Boston (2001b)

HMSO: The Health and Safety (Display Screen Equipment) Regulations, Statutory Instrument, No. 2792. HMSO, London (1992)

HMSO: Data Protection Act. HMSO, Norwich (1998) 
HMSO: Disability Discrimination Act. HMSO, London (2005a)

HMSO: Education (Pupil Information) (England) Regulations, Statutory Instrument (S.I.) No.1437. HMSO, Norwich (2005b)

ISB, Homepage Information Standards Board (2009), http: / /www. escs-isb.org . uk/ (accessed October 8, 2009)

Ip Tsang, B.C.H., Lee, S.Y.F.: Implementation of the school administration and management system: a Hong Kong experience. In: Fung, A.C.W., Visscher, A.J., Barta, B.Z., Teather, D.C.B. (eds.) Information Technology in Educational Management for the Schools of the Future, pp. 83-89. Chapman and Hall, London (1997)

MIAP, Unique Learner Numbers (2009), http: / /www.miap.gov.uk/products/uln/ (accessed October 8, 2009)

Nolan, C.J.P., Ayres, D.A.: A Developing a good information system for schools: the New Zealand experience. International Journal of Educational Research 25(4), 307-321 (1996)

Nolan, C.J.P., Ayres, D.A., Dunn, S., McKinnon, D.H.: Implementing computerised school information systems: case studies from New Zealand. International Journal of Educational Research 25(4), 335-350 (1996)

Nolan, C.J.P., Lambert, M.: Information systems for leading and managing schools: Changing the paradigm. In: Nolan, C.J.P., Fung, A.C.W., Brown, M.A. (eds.) Pathways to Institutional Improvement with Information Technology in Educational Management, pp. 72-85. Kluwer Academic Publishers, Boston (2001)

O'Mahony, C.D.: Key functions for integrated school IS management. In: Fulmer, C., Barta, B., Nolan, P. (eds.) The Integration of Information for Educational Management, pp. 105117. Felicity Press, Maine (1998)

Research Machines: Integris homepage (2009),

http: / /www .rm.com/shops/rmshop/product. aspx?cref=PD550381 (accessed October 8, 2009)

SERCO: CMIS Homepage (2009),

http: / /www. sercolearning.com/schools/products/mis.htm

(accessed October 8, 2009)

SIFA: Systems Interoperability Framework Association Homepage (2009), http: / /www. sifinfo.org/uk/ (accessed October 8, 2009)

ScholarPak: ScholarPak homepage (2009), http: / /www. scholarpack. com/ Partners / scholarpack (accessed October 8, 2009)

Schoolbacs: Schoolbacs homepage (2009), http: / / www. schoolbacs.co.uk/index.php (accessed October 8, 2009)

Selwood, I.D.: The development of ITEM in England and Wales. In: Barta, B.Z., Telem, M., Gev, Y. (eds.) Information Technology in Educational Management, pp. 85-92. Chapman hall, London (1995)

Selwood, I.D.: Information technology in educational management in schools in England and Wales: Scope progress and limits. Doctoral Thesis, Birmingham: University of Birmingham School of Education (2004)

Selwood, I.D.: Primary school teachers' use of ICT for administration and management. In: Tatnall, A., Visscher, A., Osorio, J. (eds.) Information Technology and Educational Management in the Knowledge Society, pp. 11-22. Springer, New York (2005)

Strickley, A.B.: Factors Affecting the Use of MIS as a Tool for Informing and Evaluating Teaching and Learning in Schools. In: Education and Information Technologies, March 2004, vol. (1), pp. 47-66. Kluwer Academic Publishers, Boston (2004) 
Strickley, A., Allen, S.: The role of the school MIS in pupil transfer in England. In: Tatnall, A., Visscher, A., Finegan, A., O’Mahony, C. (eds.) Evolution of Information Technology in Educational Management, pp. 129-142. Springer, Boston (2008)

Strickley, A.B.: The use of MISs in Birmingham primary schools. VDM Verlag, Germany (2009a)

Strickley, A.B.: A gateway to educational services: the challenge of parental engagement through web-enabled channels (2009b),

http: / /www. wcce2009.org/proceedings/index.html (accessed October 8, 2009)

Visscher, A.J., Bloemen, P.P.M.: CSIS usage in school management: A comparison of good and bad practice schools. In: Nolan, C.J.P., Fung, A.C.W., Brown, M.A. (eds.) Pathways to Institutional Improvement with Information Technology in Educational Management, pp. 87-97. Kluwer Academic Publishers, Boston (2001)

Visscher, A.J., Wild, P., Smith, D.: The results of implementing SIMS in English secondary schools. In: Selwood, I.D., Fung, A.C.W., O’Mahony, C.D. (eds.) Management of Education in the Information Age: the Role of ICT, pp. 33-45. Kluwer Academic Publishers, Dordrecht (2003)

WAI: Web Accessibility Initiative homepage (2009), http://www.w3.org/ (accessed October 8, 2009)

Samuel, W.: PASAPP home page (2009),

http: / /www. wautonsamuel.co.uk/Pasapp. aspx (accessed October 8, 2009)

Wild, P., Walker, J.: The commercially developed SIMS from a humble beginning. In: Visscher, A.J., Wild, P., Fung, A.C.W. (eds.) Information Technology in Educational Management, pp. 19-38. Kluwer, Netherlands (2001) 